advantages" to other patients and the general public. Perhaps they'll put in a word for wrist restraints as well, while they are at it.

It would be hard to find a psychiatrist who would not find something of interest in this book, although the price may deter private buyers.

\section{DAVID GOLDBERG}

Biomechanics of the Spine: Clinical and Surgical Perspective. Edited by vK GOEL AND JN WEInstein. (Pp 295; Price: Not Stated). London, Wolfe Publishing Ltd, 1990. ISBN 0-8493-6649-6.

This book, edited by a basic engineer in biomedical engineering and an osteopath, has been written to provide information for basic researchers and clinicians alike, and to make the practising surgeon and clinician aware of the large amount of biomechanical research that has gone into the spine in recent years. It should be more correctly called "Biomechanics of the Lumbar Spine"; there is little on the thoracic spine and nothing on the cervical spine. Eighty per cent of Western population at some time in their life will have an acute episode of low back pain which will cause a varying amount of debility and loss of time from work. They also review critically many of the "received" truths about the treatment of the lumbar spine and analyse the effects of exercises, orthoses and spinal stabilisation in the laboratory setting. They remind the reader that in the United States there are 200,000 lumbar laminectomies performed a year, with a poor outcome (published) of $10-20 \%$. Clearly, if one in five operations do not produce a good result, radical thinking has to go into the subject.

There are ten chapters and 280 pages. The line diagrams are clear and demonstrate the points well. The chapter on the anatomy of the lumbar spine is good. It is clear that their own particular research interests are on the biomechanics of the intact ligamentous spine and the biomechanics of the operated spine. These are perhaps the largest and most detailed chapters in the book. In contrast, the chapter on the mechanism of pain is five pages long and does not include all the most recent thoughts on the mechanisms of pain production, for example they quote Melzack and Wall but do not refer to the most recent edition of Wall's book and the current thoughts on pain.

The descriptions of the surgical approaches are a very good basic introduction to the clinician who is about to refer his patient for such a procedure. It demonstrates how rapidly the field of spinal surgery is moving and, to those who qualified more than ten years ago, shows how many other procedures beyond a midline laminectomy are available for lumbar spine surgery.

The role of fusion in disc disease is well reviewed. They point out that many people are subject to a fusion as well as a disectomy for lumbar disc disease and show that there is no good clinical evidence that the fusion is the ideal approach in disc disease, rather the fused segment may cause secondary deterioration at disc levels above and below the fused segment.

The treatment of idiopathic scoliosis is discussed and this is the only excursion from the lumbar spine.

The biomechanics are well described and they limit the mathematics to appendices so that the average clinician does not go to sleep immediately he begins to read the chapter. ALAN CROCKARD

Current Practice of Clinical Electroencephalography 2 nd Edition. Edited by DD DALY AND TA PEDLEY. (Pp 824; Price: \$102.00). New York, Raven Press, 1990. ISBN 0-88167-635-7.

Since the first edition of this book appeared in 1979 there has been notable progress in the field and the literature has burgeoned. Clearly an over-view of the subject is now required and this book provides it admirably. It is approaching twice the length of its predecessor, the number of chapters and contributors likewise. The scope is appropriately increased to include sections on intracranial recording, monitoring, both long-term and intra-operative, sleep, evoked responses of the main modalities including a chapter specifically on multiple sclerosis

There has been another change, the first edition was largely aimed at those in training, particularly with the American Boards in mind. This edition takes a much wider view with the editors stating modestly that, "it should also serve as an initial reference source for EEG practitioners". The succinct introduction by John $R$. Knott one of the most notable teachers in the field, acts as a signpost not only as to what it contains but also how the beginner may direct his efforts. In this context the two chapters on an orderly approach to the normal and the abnormal tracings are noteworthy. Then follows chapters which form the basis of ordinary EEG practice, on epilepsy, focal and generalised brain disorders, coma and death. They present a review often with a valuable critique of the literature. An epilogue by the editors might usefully have been longer but perhaps this reflects their exhaustion at having completed excellently a monumental task.

The standard of production is good as would be expected from these publishers. The quality of paper used to provide many well produced illustrations mean that the tome weighs 3 kilograms! It represents value for money and is warmly recommended.

DF SCOTT

Pathophysiology of Development and Rupture of Cerebral Aneurysms Supplement 48. By HANS-JAKOB STEIGER. (Pp 57; Price: DM 77, Subscribers to "Acta Neurochirurgica DM 70). 1990. Wien, Springer-Verlag. ISBN 3-211-82192-9.

This excellent monograph briefly reviews the current theories of causation of aneurysms and develops Dr Steiger's concept of aneurysmal pathophysiology derived from the experimental work undertaken by himself and others and discusses the part played by various theoretical considerations.

This book is essentially for researchers in arterial flow patterns and he gives a detailed analysis of the flow patterns of non-Newtonian fluid and demonstrates the appropriateness of Reynold's qualification of Poiseuilles's equation under experimental conditions. Dr Steiger develops the experimentally derived hypothesis that the angle between the feeding artery and the aneurysm axis follows certain rules which determine the direction of the aneurysm fundus. He continues with a geometric analysis of the relationship between aneurysm and the parent artery in patients, both angiographically and intraoperatively and concludes that there is a set of typical angles between different cerebral arteries and aneurysmal axes. Typically, these occurred between the terminal carotid, its branches and the aneurysmal axes but there was less congruence between aneurysms affecting the anterior communicating and middle cerebral artery complexes. Whilst the author questions whether the risk of rupture of an aneurysm is proportional to the surface area or volume of an aneurysm, interestingly he makes no mention of the natural history of giant aneurysms.

Dr Steiger concludes by postulating a series of events in the formation of aneurysms and their eventual rupture. He describes the nitial high shear stresses at the apices of bifurcations: further growth occurs by passively yielding to the blood pressure. Focal weakness of the aneurysmal wall may lead to a compensatory bulge or loculus, and finally he discusses the possible mechanical effects of turbulence and reflection of the pulse wave within the arterial tree.

The illustrations are clear although some of the diagrams are difficult to interpret.

This well written monograph provides an essential basis for further research into aneurysmal evolution and flow phenomena within aneurysm and their parent vessels. MICHAEL R GOODING

Motor Disturbances II (2nd Congress of the International Medical Society of Motor Disturbances-Rome, Italy 2-4 June 1988). Edited by A BERARDELLI, $R$ BENECKE, $M$ MANFREDI AND CD MARSDEN. (Pp 440; Price: $£ 50.00)$. Sidcup, Harcourt Brace Jovanovich Ltd. 1990. ISBN 0-12-089445-9.

This book contains an edited selection of papers delivered at the second Congress of the International Medical Society of Motor Disturbances, held in Rome in 1988. The main themes of the Rome congress were cortical stimulation, akinesia, the neurophysiology of cranial movement disorders, weakness and upper motor neurone involvement and neuro-imaging in motor disturbances.

The book opens with an excellent chapter by Rothwell, Day, Thompson and Marsden from the MRC Human Movement and Balance Unit in which the physiology of electrical and magnetic stimulation of the human brain is explained. Bartholow in 1874 was the first to demonstrate electrical excitability of the human cortex in a woman who had an open scalp ulcer over the parietal bones as a result of friction from a piece of whalebone in her wig. The ulcer had eroded the skull over a two inch diameter and the pulsations of the brain were clearly seen. Bartholow was able to demonstrate the insensibility of the dura and brain substance to the insertion of needles and that stimulation led to movements of the contra lateral side of the body. The strongest stimuli, however, caused the patient to have an epileptic seizure! 
In 1980 Merton and Morton at the National Hospital used a single high voltage electric shock applied to the scalp over the motor strip to record the speed of transmission of impulses through central motor pathways. This has now been replaced by the magnetic stimulator which is relatively painless. A further six well written chapters on the physiological applications of magnetic stimulation and its use in the investigation of neurological disorders, to my mind these formed the most useful section of the book.

One curious feature of the book which is largely concerned with the physiology of the motor system is the interlarding of disparate chapters on subjects such as Lisuride intravenous infusions in the on-off effect, autoantibodies against Gaba synapses in the stiff person syndrome, a general clinical review of cranial movement disorders, the effect of L-Dopa on Segawa disease and the actions of TRH-T on upper motor neurone syndromes. Good as some of these chapters are, I feel it would have been wiser for the editors to exclude them and restrict the book purely to motor physiology. Similarly while I can understand the rationale behind including the section on neuro imaging with PET, better accounts of this are available in other recent Movement Disorder texts.

This book will find an enthusiastic if limited readership and will be remembered best for the early chapters on cortical stimulation with the magnet. The delay in publication and the relative dearth of data not published elsewhere are drawbacks which could have been avoided.

AJ LEES

The Practical Management of Spasticity in Children and Adults. By $M$ B GLenN and J Whyte. (Pp 326; Price $£ 25.57$ ). Beckenham, Lea \& Febiger (UK) Ltd. 1990. ISBN 0812112970.

Rehabilitation of people with neurological disease is particularly challenging because of the complex interactions between multiple impairments and the resulting disabilities. To achieve the best outcome, the neurologist should work closely with colleagues from other medical and therapy specialties as part of a team, adopting an holistic approach with each patient at its centre.

On the face of it therefore, a book devoted exclusively to spasticity might appear inappropriate. Coexistent cognitive sensory and sphincter impairments cannot be ignored, even if it were possible to separate cerebellar and extrapyramidal disorders from the "upper motor neurone" syndrome. Furthermore, within the latter, negative features such as weakness have functional consequences often greater than the positive intrusion of spasticity. Nevertheless spasticity is extremely common and often conspicuous, hence it is tempting to treat it just because it is there.

The editors of this book have avoided these pitfalls, assembling a well-balanced team of authors including surgeons, therapists and physicians mainly from Boston. The idea for the book grew out of a teaching course of the same title, and the emphasis is on assessment and treatment approaches. There are separate introductory but comprehensive chapters on neurophysiology, neurophysiologic testing, and objective measurement in spasticity, and an interesting discussion on the mechanisms of learning and relearning motor skills. Positioning, physical modalities, upper limb splinting, lower limb orthotics, anti-spasticity drugs, nerve blocks, neurosurgical approaches (including intrathecal drug infusion), and orthopaedic procedures each have a chapter. Two final chapters illustrate differences in management of multiple sclerosis and the after effect of head injury.

This is an excellent review of all available options, including sound practical advice and honest assessment of their likely efficacy. The volume is well edited and produced, and thoroughly referenced. Its practical emphasis and reasonable price should make it all the more attractive to anyone regularly engaged in the practical management of spasticity in children and adults. Strongly recommended.

RJ HARDIE

Musculoskeletal Medicine: The Spine. By L BURN AND J K PATERSON. (Pp 301; Price: Dfl 175.00, US\$ 90.00, UK $£ 50.00$ ). Dordrecht, Kluwer Academic Publishers Group. 1990. ISBN 0-7923-8913-1.

This is an unusual book written by two Presidents of the British Association of Manipulative Medicine. They take a sceptical look at the knowledge on which we base our clinical management of spinal pain, and review current treatment. It is a difficult book to read, much of it in short paragraphs, which makes it rather like reading Wittgenstein without the fun. The book is in two halves, Part One-Basic Considerations devoted to relevant epidemiology, relevant anatomy, relevant physiology and so on; Part TwoClinical Application includes basic case analysis, clinical presentation and "tiers of management".

The first half sets out to look at what we know or rather don't know about spinal pain supported by selected excerpts from favoured texts and replete with references-some obscure, for instance "in the Seventh Congress of Federation of International Manual Medicine (unpublished)". In disheartening fashion the authors knock down the precepts of deterministic medicine using highly selected scientific data. Thus the truth emerges that we know virtually nothing about the origin of spinal pain, and clinicians are not entitled to make a diagnosis. Thus empiricism rules. Along the way the authors demonstrate their wide breadth of knowledge from mechanoreceptors to psychotherapy. Unfortunately, although there is much information it is difficult to learn from these two teachers, partly because of the presentation. It is a shame, for instance, that in a section on complicated anatomy there is not one illustration, and in the whole book there is only one diagram and no radiographs. The latter omission is not surprising; the authors claim, with some justification, "to ascribe (vertebral) pain to degenerative changes (on x-ray) is clearly a clinical error". The gist of all this is to prove that the "clinician is empiricist, diagnostic and therapeutic, like it or not". Unfortunately they blow it all with their second half. Here they move from using scientific method to destroy current shibboleths to create their own equally unsustainable practice. The authors are in danger of throwing away the neurological baby with the bath water. Although an empiricist approach may well be the best for the vast numbers of patients presented to these doctors with spinal pain, surely a little neurological determinism wouldn't go amiss in some cases. Orthodox examination is to be regarded as "accessory". Reflexes, for instance, have little value. The authors assess spinal pain by alternative methods-“"skin pinching", "muscle guarding", "trigger points" and "segmental sagittal lateral spinous process pressure". All these techniques are searching for localised spinal tenderness but how this influences case management is not clear. The last section describes 30 different types of therapy in noteform from surgery to acupuncture, and is at its strongest when describing behavioural and cognitive approaches to back pain.

The authors have approached their topic in a polemical way which will not be to the liking of many. It is a challenging read which I find difficult to recommend. However, I did like their alternative title, which I am sure would have sold more books-"Rheumatology without the lymphocyte".

CG CLOUGH

Localization in Clinical Neurology. 2nd edition. Edited by P W BRAZIS (Pp 509 Illustrated; Price $£^{47.50)}$. Edinburgh: Churchill Livingstone, 1990. ISBN 0-316-10743-3

In the preface to the first edition of this book the authors ask the question "Is there still a role for clinical localization in neurological practice in the CT era?" I feel they provide an unequivocal answer in the second edition of Localization in Clinical Neurology. The first edition of the book published in 1985 was well received and I am sure this reception will be accorded to the second edition.

I have greatly enjoyed reading this book for it provides an extensive revision course in clinical applied neuroanatomy. The authors direct this edition at what they term "front line" clinicians and towards those training in neurology, neurosurgery and related fields. I think to these people, and also more senior members of the profession whose neuroanatomy is a little distanced by time, the book will be very attractive. Particularly useful are the opening chapters on the neuroanatomy and localisation of peripheral nerve lesions, with a succinct well illustrated account of lesions affecting peripheral nerves. These chapters provide an access to clinical answers not easily found in the larger texts on peripheral nerve injury. Plexus lesions are considered separately and the authors arrange their further chapters topographically dealing with spinal cord, cranial nerve and other intracranial areas of localisation.

What is new in this book? To this reviewer the Flynn phenomenon and Flouren's law were discovered in the chapter on the ocular motor system, otherwise nothing is new, but what is here is very carefully put together and made very enjoyable and instructive reading. There is very little to criticise. Perhaps the account of the syndromes of anterior spinal artery insufficiency should include, or refer to, acute demyelinating transverse myelitis. Transverse myelopathy of acute onset is discussed in its relationship to trauma tumour, vascular disorders and multiple sclerosis but is not mentioned under "Mul- 